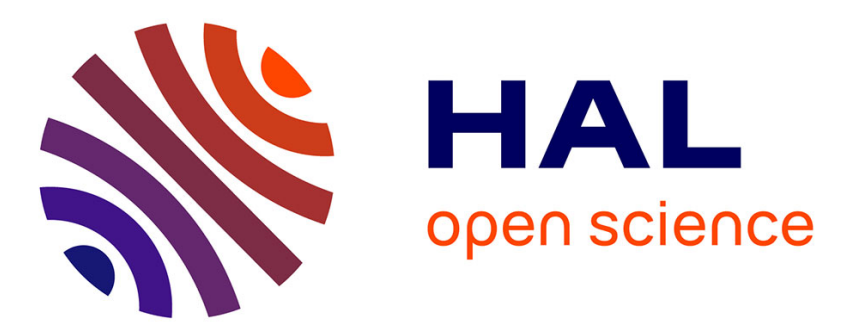

\title{
Retrait des terres, aménagement rural et agriculture dans les zones défavorisées: une question pour les Pyrénées
}

\author{
Monique Barrue-Pastor
}

\section{To cite this version:}

Monique Barrue-Pastor. Retrait des terres, aménagement rural et agriculture dans les zones défavorisées: une question pour les Pyrénées. Revue Géographique des Pyrénées et du Sud-Ouest, 1989, Pyrénées, 60 (2), pp.258-271. 10.3406/rgpso.1989.3157 . hal-02865945

\section{HAL Id: hal-02865945 \\ https://hal.science/hal-02865945}

Submitted on 12 Jun 2020

HAL is a multi-disciplinary open access archive for the deposit and dissemination of scientific research documents, whether they are published or not. The documents may come from teaching and research institutions in France or abroad, or from public or private research centers.
L'archive ouverte pluridisciplinaire HAL, est destinée au dépôt et à la diffusion de documents scientifiques de niveau recherche, publiés ou non, émanant des établissements d'enseignement et de recherche français ou étrangers, des laboratoires publics ou privés. 
Retrait des terres, aménagement rural et agriculture dans les zones défavorisées : une question pour les Pyrénées

Monique Barrué-Pastor

\section{Citer ce document / Cite this document :}

Barrué-Pastor Monique. Retrait des terres, aménagement rural et agriculture dans les zones défavorisées : une question pour les Pyrénées. In: Revue géographique des Pyrénées et du Sud-Ouest, tome 60, fascicule 2, 1989. Pyrénées. pp. 258-271; doi : https://doi.org/10.3406/rgpso.1989.3157

https://www.persee.fr/doc/rgpso_0035-3221_1989_num_60_2_3157

Fichier pdf généré le 06/04/2018 
décentralisation: absence jusqu'en 1987 de «Programmes d'intérêt général " et de "prescriptions particulières» élaborées au niveau des massifs. Toujours est-il que dans une montagne qui s'est encore dépeuplée entre 1962 et 1986 (-10\% malgré les revirements démographiques enregistrés après 1975), dans un espace où la population agricole devient partout minoritaire, où la "rurbanisation" progresse en même temps que le nombre des résidences secondaires, trois intérêts majeurs s'affrontent et transforment l'aménagement de la montagne en un véritable enjeu national: l'un attaché au droit coutumier et à une activité agricole déliquescente, l'autre liée à l'aménagement touristique, le troisième confondu avec la préservation du patrimoine, qualifićc par l'auteur "d'intérêt public nouveau ».

On regrettera que cette thèse n'aborde pas le problème central que pose à tous - populations, élus locaux, pouvoirs publics... - l'avenir particulièrement incertain de la montagne pyrénéenne. On sait en effet que l'argument de la survie économique des populations concernées est souvent opposé à celui de la protection de l'environnement. A. Etchelecou démystifie cependant le prétendu succès économique de certaines opérations d'équipement lourd de la montagne. Il reconnaît aussi que, globalement, les stations de ski ont permis un enracinement des populations, même si elles ont favorisé l'élimination d'une agriculture fragilisée. Il propose surtout qu'une meilleure rationalité économique préside à des choix d'investissements décidés trop souvent à la légère, sous la pression du pouvoir local et des groupes qui gravitent autour de lui.

Quels qu'aient été, à ce jour, les aménagements réalisés, les perspectives démographiques pour le début du $\mathrm{XXI}^{\bullet}$ siècle ne sont guère optimistes. La population diminuera un peu partout et supportera le poids plus lourd du troisième âge. Selon l'auteur, "l'avenir du peuplement demeurera guidé par l'attractivité de l'espace " et cette dernière suppose que \&l'on tende vers un aménagement optimal " défini en fonction de trois principes : "la préservation du patrimoine naturel et culturel", la "non altération de la diversité biologique", la "prise en compte (véritable) du risque économique " contre une socialisation systématique des coûts, pertes et autres déficits... Ces réformes qui s'opposeraient au compartimentage de l'espace comme à l'absolutisme des pouvoirs centraux et locaux, qui permettraient à un contre-pouvoir associatif d'éclore et de se faire entendre, seront-elles entreprises? Rien de moins sûr!

Guy Di Méo.

\section{RETRAIT DES TERRES, AMENAGEMENT RURAL ET AGRICULTURE DANS LES ZONES DEFAVORISEES : UNE QUESTION POUR L.ES PYRENEES}

De premières analyses de déprise agricole et rurale dans plusieurs communes de Midi-Pyrénées, permettent d'inférer une probable aggravation 
du phénomène étant donné le nombre des exploitants âgés sans successeurs. Ce processus qui a atteint $d$ abord les zones les plus défavorisées, entre autres les zones de montagne, est en train de s'étendre à près de $70 \%$ de la France rurale; ces zones sont dorénavant dites "fragiles", comme en témoigne une carte publiée récemment par le ministère de l'Agriculture et de la Forêt, à l'occasion des contrats de plan Etat-région (fig. 2). L'objectif premier de la politique d'aménagement du territoire est devenu la lutte contre la «désertification » entendue dans le sens de dépopulation et de déclin économique, notamment d'abandon de l'activité agricole.

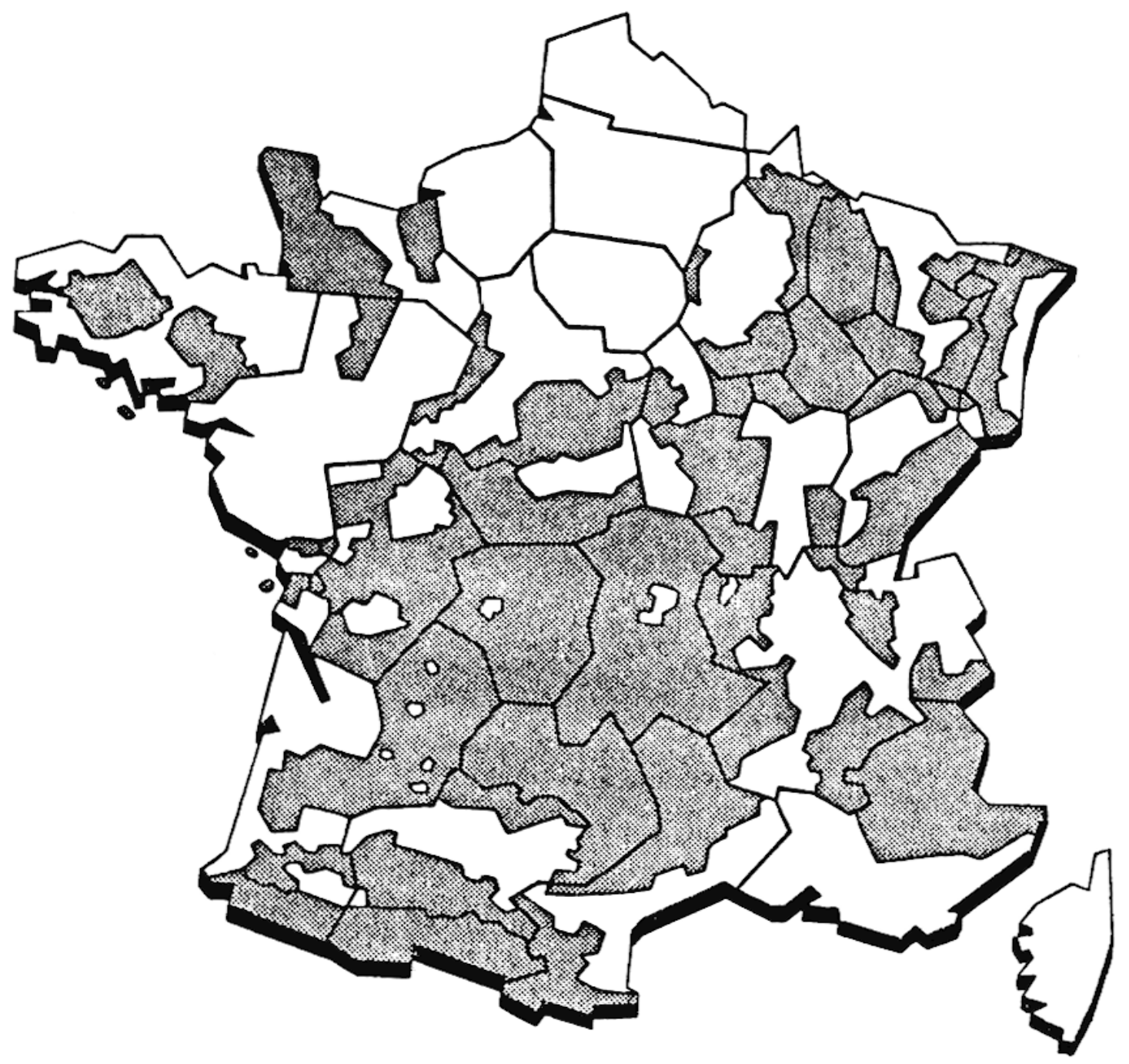

FIG. 2

Extension des zones rurales fragiles en France.

Elles sont délimitées dans la perspective des «Programmes d'aménagement concerté du territoire " des contrats de plan Etat-Région. (Source : ministère de l'Agriculture et de la Forêt). 
Dans un tel contexte, la publication d'un décret ministériel relatif au retrait des terres arables (5), souvent désigné comme le «gel des terres", impressionne vivement non seulement les milieux agricoles déjà traumatisés par le système des quotas, mais aussi le grand public pénétré d'une culture dans laquelle la terre reste une mère nourricière et pour qui le défrichement est un progrès de civilisation. Depuis 1980, l'Europe se trouve confrontée à la rupture d'équilibre des marchés des principaux produits agricoles: la consommation intérieure européenne est devenue inférieure à la production intérieure; c'est-à-dire que la capacité de production est supérieure aux débouchés sur le marché solvable. C'est pourquoi il est question de production agrico!e « excédentaire » et que l'objectif de la politique agricole européenne commune est devenu la réduction de la production agricole. Dans les années 1984-1985, les quotas (6) laitiers ont été la première mesure prise pour limiter le déve'oppement du vo'ume des productions agrico'es. Le retrait des terres, c'est-à-dire la mise hors culture d'une certaine superficie de terres arabies, s'inscrit dans la même perspective, mais ni avec la même connotation sociale, ni avec le même effet territorial. Il fait partie d'un ensemble de mesures "sociostructurelles » proposées à partir de janvier 1989 aux agriculteurs : reconversion, extension, aide aux revenus, cessation anticipée d'activité (7).

Les inquiétudes se multiplient donc, bien qu'il soit trop tôt pour évaluer les conséquences de ces mesures. Une première évaluation de leurs effets peut d'ores et déjà sortir de la connaissance fine de certaines de ces zones - la zone défavorisée pyrénéenne dans le cas présent - et de l'analyse des premiers dossiers qui sont parvenus au 30 avril 1989 (8), date de clôture de la première campagne, au Centre national pour l'aménagement des structures des exploitations agricoles chargé de payer les primes. Il s'agit donc d'identifier les questions que pose l'app'ication de ces mesures et leurs conséquences possibles sur l'aménagement et le développement rural, qu'elles soient d'ordre écologique, social ou économique.

(5) Le Conseil des ministres des Etats membres de la Communauté économique européenne a adopté lors de sa session du 12 février 1988, à Bruxelles, une recommandation visant le retrait des terres agricoles. En France, c'est le décret du 18 novembre 1988 qui détermine les modalités d'application de cette mesure liée à de la politique agricole commune dont la première campagne s'est étendue de l'automne 1988 au printemps 1989.

(6) Quota : système de limitation quantitative de la production agricole qui répartit (avec plus ou moins d'équité) le droit de produire entre les producteurs.

(7) Voir, entre autres, à leur propos D. RomÉAs, "Le gel des terres et après ? ", Purpan, $4^{\circ}$ trimestre, 1988, p. 165-170.

(8) Au 30 avril 1989, 450000 hectares avaient été mis en jachère, mais surtout en Allemagne fédérale et Italie (35000 agriculteurs concernés pour 300000 hectares), d'après M. Ray MACSHARRY, commissaire européen à l'agriculture. En France, malgré les réticences manifestées autant par le ministère de l'Agriculture que par une majorité d'agriculteurs qui tiennent les primes pour insuffisamment attractives (et malgré le retard de la diffusion de l'information qui s'en est suivi, 6000 dossiers avaient été inscrits pour une superficie de 70000 hectares environ; 1002 ont été reçus, aboutissant au retrait de 15708 hectares (en MidiPyrénées, 135 dossiers (dont 39 en Haute-Garonne (maximum) et aucun dans les Hautes-Pyrénées (minimum) pour une superficie de 1509 hcctarcs). Mais le processus pourrait s'accélérer lors de la prochaine campagne. 
Le retrait des terres est pratiqué depuis longtemps aux Etats-Unis (années 1960) et plus récemment en République fédérale d'Allemagne et en Grande-Bretagne; mais ces expériences étrangères (9) apportent plus de questions que de réponses. D'où notre souci d'une évaluation des effets pervers que pourraient avoir ces mesures sur les zones défavorisées déjà menacées en France par la désertification et qui pourraient bien être les premières victimes de ces mesures malgré les objectifs affichés. Car le choix du retrait des terres arables pour diminuer la production agricole, sans qu'il y ait remise en cause des processus d'intensification, entre autres, de l'usage massif d'engrais, ne va pas forcément remettre en cause la logique productiviste ni la concentration de la production sur les meilleures terres; l'expérience américaine enseigne que si 33 millions d'hectares ont été "gelés " depuis 1983, la baisse de la production céréalière n'a été que de $1 \%$.

La perspective d'application de ces mesures fait surgir de nouveaux antagonismes, notamment entre plaine et montagne, entre pôles de productions agricoles et zones rurales en déclin ou marginalisées. De nouvelles contradictions apparaissent entre l'objectif affiché de l'aménagement du territoire, lutter contre les processus de désertification, et les objectifs de la politique agricole commune qui vise à réduirc les excédents par la diminution des surfaces cultivées.

\section{Enjeux contradictoires plus que double discours.}

Avant de présenter le dispositif d'application du gel des terres et de proposer de lui donner un sens dans l'histoire des réformes de la politique agricole commune, il convient de resituer ce décret dans le contexte socio-économique plus général de l'aménagement du territoire dominé par la continuité des processus de déprise agricole et d'enfrichement, pour ne pas dire de désertification. Il faut aussi souligner que ces nouveaux instruments de pilotage de l'agriculture et de l'espace rural sont disposés dans une période marquée par des discours alarmistes antagoniques. L'un, d'essence "environnementaliste", porte sur la déprise agricole, la désertification et la dégradation des paysages ruraux d'une France dite "profonde ». L'autre, de nature "économiste", porte sur la surproduction, la saturation des marchés et l'accroissement des rendements, présentés comme des témoignages d'un "excédent de terre " de plus en plus considérable et que des experts évaluent entre 5 et 15 millions d'hectares (d'ici la fin du siècle), selon la capacité de la Communauté économi-

(9) Ce genre de comparaison nécessiterait une étude qui n'a pas été faite. Nous nous référons ici aux conclusions de François Hourller, Le gel des terres communautaires, ESOPE $n^{\circ} 459$, mai 1988, du document de travail sur Les programmes de restriction de l'utilisation des terres agricoles dans les pays de I'O.C.D.E., Paris, octobre 1988, de Jacques Blanchet et Jean-Claude SouRIE, Le retrait des terres agricoles. Mesures de circonstance ou solution d'avenir?, I.N.R.A., Paris-Grignon décembre, 1988. 
que européenne d'en terminer avec ses importations de produits de substitution aux céréales et à freiner celles de soja. Dans ce contexte, on pourrait supposer que la question fondamentale serait de savoir comment concilier, à l'échelle de la Communauté, le déclin du monde rural et le dépérissement de certaines régions avec la diminution des productions et des surfaces agricoles. L'accé ération de l'enfrichement peut-il être une réponse à cette question? Mais, d'autre part, non seulement les cau. ses et les conséquences locales de la déprise agricole des zones défavorisées ne cessent de se multip!ier, mais les politiques sectorielles nationales et européennes accentuent de jour en jour leur déséquilibre et leur fragilité.

D'un autre côté, le bilan de 25 ans d'aménagement du territoire en France, arrêté à Paris le 25 février 1987, a conclu à la nécessité d'une mise en œuvre rapide d'actions en faveur du maintien de l'activité agricole dans les régions les plus sensibles, alors définies comme des "zones rurales sinistrées". "La France ne peut plus se permettre après avoir accepté les friches industrielles, d'accepter les friches rurales, ni d'accrô̂tre les coûts de concentration et de désertification $*(10)$.

\section{Rétraction des terres agricoles, enfrichement, extensification : un processus continu de déprise agricole.}

a. Trois causes fondamentales demeurent: le problème foncier, le vieillissement de la population et l'hétérogénéité professionnelle et sociale.

Les propriétaires fonciers agriculteurs sont devenus minoritaires: ils ne représentent plus que 20 à $50 \%$ des propriétaires selon les communes dans les Pyrénées centrales; ils continuent à être cependant les principaux utilisateurs de l'ensemble des terroirs de montagne. Ils ont perdu le contrôle du foncier et de la gestion du territoire avec les règlements de succession et l'arrivée de nouveaux ruraux. Ils ont aussi, très souvent, perdu le contrôle du pouvoir local : non seulement les maires mais aussi les conseillers municipaux agriculteurs sont devenus rares.

Le nombre des agriculteurs âgés qui refusent de vendre et surtout de louer - avec bail - les terres pour un usage agricole aggrave la précarité du fonctionnement des exploitations agricoles et obère leur avenir.

L'hétérogénéité de la société rurale est enfin devenue, de deux manières, un facteur supplémentaire de fragilisation. Celle-ci vient de pressions intérieures qui tiennent à la diversification des pratiques, des projets, des profils des actifs agricoles et qui sont à l'origine de nouvelles contradictions internes à l'agriculture, ainsi le poids des exploitants sans successeurs.

(10) «Forum : 25 ans d'aménagement du territoire ", Paris, 25 février 1987. 
Elle vient aussi de l'environnement social local, dans lequel les agriculteurs, devenus minoritaires, sont les premières victimes des contradictions qui, en l'absence de planification globale, caractérisent toute option d'aména. gement du territoire; les objectifs de conservation et de développement, en particulier, sont rarement appariés.

b. Trois conséquences sur le territoire marquent ce processus de déprise agricole; elles sont caricaturales en zone de montagne.

On observe à la fois la rétraction des terres agricoles et l'extensification; cela se manifeste non seulement par la diminution des superficies utilisées et par la diminution de l'entretien, mais par une délégation des choix de gestion de l'homme à l'animal; c'est le cas, par exemple, du passage des prés de fauche entretenus et fumés à un pacage libre dont la seule gestion consiste dans les choix effectués par les animaux en quête de fourrage.

On mesure, d'autre part, l'appauvrissement de la fertilité des sols, conséquence du défaut d'entretien et de la généralisation de la pratique du système de cueillette, qui pourrait aller jusqu'à la remise en cause, à moyen terme, de l'activité pastora!e de fond de vallée (11). Ce sont ici les conditions sociales $d$ accès au foncier, c'est-à-dire la précarité des locations verbales, qu compromettent la reproduction d'une ressource naturelle renouvelable: l'herbe.

On constate enfin le développement de l'enfrichement par " non gestion ». Ce défaut affecte, par exemple, les soulanes, anciennes zones de champs exposés au sud, aux sols riches mais peu profonds, aux structures foncières extrêmement exiguës; elles sont passées sans transition, et en 25 ans, d'une culture agricole intensive à un parcours extensif et libre. Dans ce cas, se développe un reboisement anarchique: pousse un bois défini comme "tout venant " sans grande valeur économique (noisetiers, bouleaux); cela accélère le processus de fermeture des paysages: les parcelles deviennent enclavées, inaccessibles; les chemins s'embroussaillent et disparaissent.

Ici, la logique agricole n'a été remplacée par aucune autre option; le reboisement anarchique ne participe pas d'une option économique de reforestation; l'abandon de l'agriculture s'accomplit donc sans qu'il y ait valorisation d'un nouveau potentiel économique basé sur la forêt. Dans ce contexte, les coûts engendrés par la désertification ne cesseront d'augmenter; nous sommes en train de vivre un mouvement de fragilisation extrême des sociétés montagnardes; le processus de diversification des sources de revenus et des systèmes d'expioitation en tant que principe d'adaptation aux contingences locales et comme moyen de résistance semble avoir atteint ses limites. Mais les conditions nécessaires au maintien d'un minimum d'activité agricole dans les zones défavorisées sont-elles compa-

(11) Voir, à ce propos, G. Balent et M. Barrué-Pastor, Pratiques pastorales et stratégies foncières dans le processus de déprise de l'élévage montagnard en vallée d'Oó (Pyrénées Centrales), RGPSO, 57, 3, 1986, pp. 403-447. 
tibles avec les dernières directives de la politique agricole de la Communauté européenne?

III. La politique agricole commune: de l'affaiblissement à la condamnation de l'agriculture montagnarde et des zones fragiles.

La première période de la politique des structures agricoles de la Communauté européenne (1978-1984) fut axée sur la notion d'entreprise économique susceptible de s'assurer un revenu de parité, c'est-à-dire comparable aux autres secteurs de la vie économique. A partir de 1985, un nouveau débat sur la réorientation de la politique des structures découlant du Livre vert (12) s'ouvrit, principalement axé sur le maintien de l'espace rural, considérant, entre autres, la nécessité pour la po:itique agricole de prendre davantage en compte la politique de l'environnement et, également, l'adaptation de l'agriculture à la nouvelle situation des marchés.

Le règlement du 15 juin 1987 modifie sensiblement les règlements antérieurs mais contient surtout deux séries de mesures nouvelles qui préconisent "l'agriculture environnementa!e» et "l'extensification ». Un nouveau grand rôle est assigné à l'agriculture: celui d'être une "activité de production qui contribue à l'entretien de l'espace naturel environnant », notamment dans les zones sensibles du point de vue de la protection de l'environnement. Or, paradoxalement, quatre de ces mesures risquent de signer l'arrêt de mort de l'agriculture des zones fragiles, notamment si elles étaient adoptées en l'état actuel de leur libellé, encore ssoumis à discussion pour certaines d'entre elles.

a. La législation sur le retrait des terres arables. Après de longs tâtonnements du gouvernement français et des commissions économiques, à Bruxelles, le dispositif de "retrait des terres arables " est paru au Journal officiel du 19 novembre 1988. Il convient d'en rappeler les principales dispositions et de souligner qu'il est expérimental jusqu'à la fin de l'année 1989, d'où l'intérêt de développer les analyses critiques.

En vertu des décisions communautaires, l'aide au retrait des terres est réservée à l'exploitant - et pas uniquement au propriétaire - qui s'engage à ne pas cultiver au moins $20 \%$ au maximum $100 \%$, dans le cas de jachères fixes (ou $30 \%$ dans le cas de jachère tournante) de ses terres arables pendant cinq années. Seules peuvent être prises en compte les terres dont on justifiera qu'elles ont été cultivées entre le $1^{\text {er }}$ juillet 1987 et le 30 juin 1988. Le bénéficiaire ne peut pas être en retraite au moment de sa demande. La superficie minimale de retrait est de un hectare d'un seul tenant, mais peut correspondre à plusieurs parcelles cadastrales. On dis-

(12) Livre vert, Bruxelles, juillet 1985. Réflexions sur les perspectives de la Politique agricole commune (P.A.C.). 
tingue donc la jachère nue et fixe de la jachère tournante (13); mais la proposition de "jachère pâturée " proposée par M. François Guillaume (14), qui laissait craindre un transfert de la production de viande vers les régions céréalières, a été rejetée. Certes, la jachère fixe ne bénéficie pas $\mathrm{d}$ une prime aussi élevée ( $1200 \mathrm{~F} / \mathrm{ha} / \mathrm{an}$ en moyenne) que la jachère tournante ( $2000 \mathrm{~F} / \mathrm{ha} / \mathrm{an}$ en moyenne) que le ministère de l'Agriculture entend privilégier (fig. 3), mais cela ne suffira pas à éviter les abandons définitifs de terre étant donné l'importance qui est accordée au reboisement et aux utilisations non agricoles (15). Au-delà de la mise en jachère des terres, c'est bien un problème de changement d'affectation des sols qui doit êtro: posé.

Ce décret a pour objectif de mettre hors culture une partie des terres agricoles. Pour une p!eine efficacité, il ne devrait s'appiiquer qu'aux terres les plus productives, mais on peut s'attendre qu'il soit utilisé dans n'importe laquelle des zones agricoles, ce qui pourrait bien en faire le règlement structurel le plus marquant qu'auront connu depuis longtemps l'agriculture et la gestion des espaces ruraux. En l'état actuel d'application de la réglementation, les moyens de contrôle des demandes restent fort limités, ce qui revient à rendre acceptable la quasi-totalité des dossiers instruits.

Il suffit en effet d'une déclaration sur l'honneur pour attester que les terres visées par la demande de mise en jachère subventionnée sont " arables", même si par ailleurs, elles sont inscrites en nature de "bois * ou de "landes» sur le cadastre. Pour savoir si ces terres sont réellement cultivées par le demandeur, une déclaration à la Mutualité sociale agricole attestant que l'intéressé est l'exploitant suffira; un bail écrit n'est pas nécessaire pour les non propriétaires. Le plan cadastral des terres mises de la sorte en jachère subventionnée n'étant pas obligatoire, il semble très difficile, au vu des dossiers, de savoir quelle réalité ils recouvrent... $\mathrm{O}_{i}{ }^{-}$les vérifications imposées par la C.E.E. ne portent que sur $5 \%$ des dossiers. Tous les abus semblent donc possibles.

b. Perspectives envisageables. Etant donné la complexité des mesures proposées, nous nous contenterons de présenter ici leurs principaux objectifs et les menaces qu'elles font peser sur les zones de montagne. En

(13) Jachère fixe : mise en jachère durant cinq années. - Jachère tournante : ici, mise en jachère d'un même pourcentage de la superficie de l'exploitation, mais selon une rotation traditionnelle, le plus souvent triennale. - Jachère pâturée: surface non cultivée, enherbée, susceptible d'être pâturée, mais, selon la directive européenne, uniquement dans le cadre d'un élevage extensif, afin d'éviter que les excédents de céréales ne se "transforment » en excédents de production de viande bovine ou ovine; il s'agit donc de pâturages permanents dont le foin pourra être coupé pour les besoins de l'exploitation.

(14) Ci-devant ministre de l'Agriculture (1986-88), ci-devant responsable national de la F.N.S.E.A.

(15) Dans chaque département de Midi-Pyrénées les préfets ont à définir le montant de la prime pour chaque petite région agricole a l'intérieur d'une fourchette imposée: (toutes zones confondues le minimum est de $1000 \mathrm{~F}, 1 \mathrm{e}$ maximum est de $2600 \mathrm{~F}$ l'hectare, par an). 


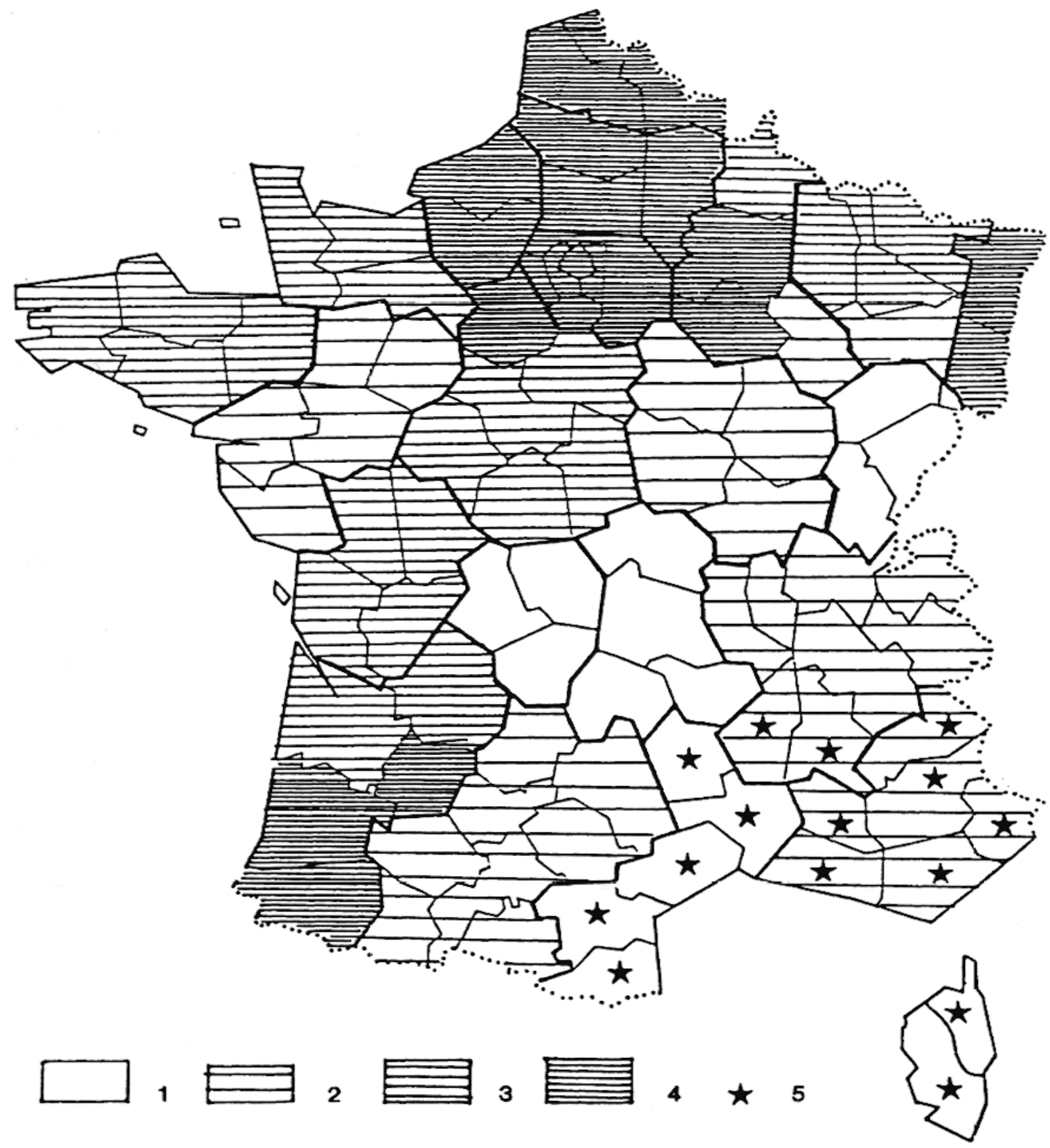

FiG. 3

Montant des primes annuelles accordées pour le retrait des terres arables en France pendant la campagne 1988-1989.

(Décret ministériel du 18 novembre 1988).

1. Primes possibles entre le minimum de $1000 \mathrm{~F} / \mathrm{ha}$ et le maximum de $1700 \mathrm{~F} / \mathrm{ha}$. - 2. 1200 à $2000 \mathrm{~F}$. - 3. 1500 à $2300 \mathrm{~F}$. - 4. 1800 à $2600 \mathrm{~F}$. 5. Zones pouvant faire l'objet d'une exclusion de l'aide. (Source : Centre national pour l'aménagement des structures des exploitations agricoles). 


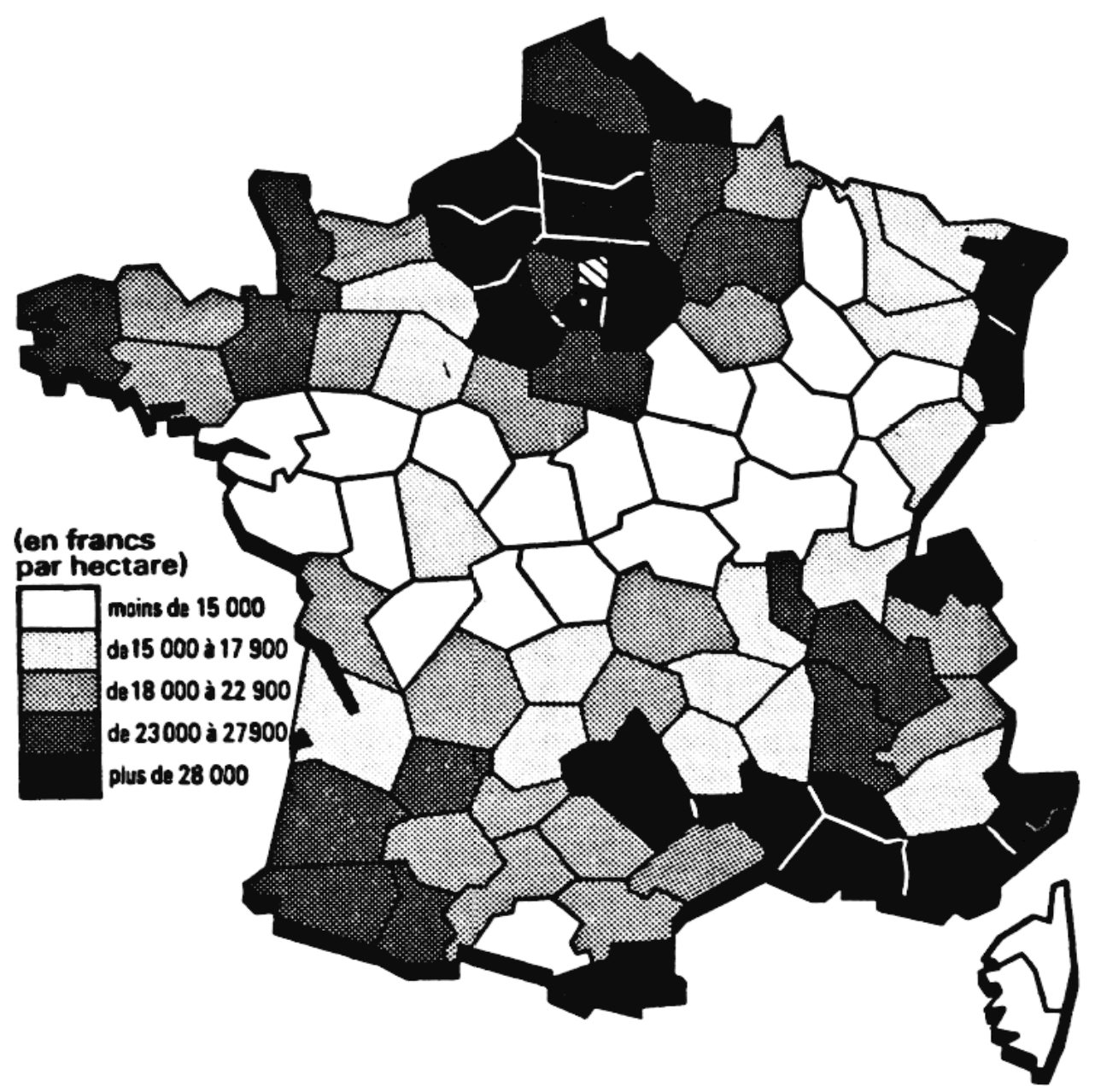

Fig. 4

Prix de vente moyens des terres labourables et prairies naturelles observés en France en 1987 par départements.

Les prix moyens s'établissent à $19800 \mathrm{~F} / \mathrm{ha}(21700$ les terres labourables et 16700 pour les prairies), en baisse de $1,8 \%$ par rapport à 1987 (ou de $4,8 \%$ en valeur réelle); la baisse cumulée, en francs constants, est de $46 \%$ depuis 1978.

(Source : Service central des enquêtes et études statistiques du ministère de l'Agriculture et de la Forêt).

fait le retrait des terres revient "à mettre de côté " (avec primes diverses) les terres agrico:es selon deux cas de figure. Ou bien toute possibilité de production agricole leur est retirée définitivement ou temporairement; une aide au boisement des surfaces agricoles est d'ailleurs instituée. Ou bien elles sont seulement mises à part pour une certaine période; dans ce cas, leur vocation productive est maintenue, soit par la jachère fixe, soit par une jachère tournante. 
Se'on la manière dont le retrait va s'effectuer, les conséquences possibles semblent très diverses, à la fois pour l'environnement et pour l'exploitation des ressources naturelles renouvelables. Si les terres sont laissées en friche, ce sera l'extension directe de l'enfrichement et la fermeture des paysages. Si les terres sont reboisées, perspective qui pourrait s'avérer très attractive selon les modalités concrètes que prendra son application, le boisement doit être conforme à la réglementation en vigueur dans la commune; s'il y a un zonage agriculture-forêt, ce qui est fort rare, il faut donc que le boisement proposé se fasse en continuité avec des boisements existants d'au moins dix hectares. Une contribution au reboisement, dont l'origine n'est pas encore signalée, devrait alléger les investissements consentis par l'agriculteur lui-même. Cette mesure pourrait pousser ceux qui sont en difficulté, et dont l'agriculture n'est pas le revenu principal, à reboiser la totalité de leur S.A.U., ce qui aurait de multiples conséquences négatives, notamment en étendant les zones dépeuplées et en compromettant l'avenir du patrimoine foncier. En reboisant sans discernement la totalité de la S.A.U., on court d'ailleurs le risque de ne produire que du bois "tout venant s sur les mauvaises terres cependant que les meilleures terres seront écartées de la production agricole.

La "diminution harmonieuse" de la surface agricole prônée par la Communauté européenne ne serait-elle pas compromise par les modalités et les rythmes d'attribution des crédits? En effet, les aides européennes se caractérisent en la matière par le retard des attributions et l'obligation de les dépenser dans des délais réduits. En conséquence, le travail de prospection et de sensibilisation des agriculteurs à la culture forestière sera malaisé sinon impossible, ce qui pourrait empêcher d'atteindre l'objectif de polyvalence des activités, où l'on envisage d'inclure un volet forestier par la reconversion partielle de la S.A.U.. En conséquence également, la répartition du quota à reboiser (par département) en lots maîtrisables par l'agriculteur (surfaces de 10 à $20 \mathrm{ha}$ ), tant au niveau de l'entretien que des moyens techniques dont il dispose, risque fort d'être rempiacée par un reboisement à la chaîne sur de grandes surfaces, laissées à l'abandon après la plantation. Ainsi, les terres extraites de la production agrico!e et reboisées risquent de gêner la survie d'une agriculture déjà extensive et en difficulté et d'accroître le processus de désertification sans pour autant créer un nouveau potentiel économique fondé sur la forêt.

Si les terres sont utilisées à des fins non agricoles, à caractère touristique ou de loisir, les terrains de jeu, de golf, de camping pourront se déve.opper ainsi que le processus d'urbanisation, reposant ainsi avec acuité la question dun aménagement global du territoire. C'est cette dernière possiblité qui intéresse ou qui préoccupe le plus les élus locaux : déjà plusieurs golfs sont projetés en Midi-Pyrénées et on compte ce genre de projet par dizaines dans l'ensemble du Sud-Ouest au printemps 1989.

Sans vou'oir préjuger des conséquences négatives de l'ensemble des mesures favorisant le retrait des terres, il semble d'ores et déjà que les 
primes accordées en compensation de l'abandon de l'agriculture soient disproportionnées avec le coût de la désertification qu'elles pourraient engendrer. La constitution d'une forme de "rente du retrait de l'agriculture " en tant que prix à payer pour en finir avec les excédents communautaires de la production agricole semble paradoxale; elle ne sert ni la cause des intérêts particuliers, ni celle de l'intérêt général (entre autre, du patrimoine foncier) à moyen ou à long terme.

c. Effets d'autres dispositions réglementaires. Il s'agit de la reconversion, de "I'extensification" et de la préretraite, dont l'esprit est le même que celui du retrait des terres. L'objectif final est de diminuer le volume des productions... ce qui ne va pas sans rappeler le premier mémorandum sur la réforme de l'agriculture dans la Communauté, comme « le plan Mansholt ". Dans le premier cas, on vise à réduire les volumes produits en excédent en incitant, par des aides financières, les agriculteurs à se reconvertir vers des productions qui ne sont pas soutenues par le budget communautaire. Dans le deuxième cas, celui de "l'extensification", on entend diminuer le volume des productions en suscitant une baisse des rendements; l'agriculture de montagne risque de subir une nouvelle concurrence : celle de zones hautement productives en céréales, qui se seront reconverties dans l'élevage pour la viande.

La cessation anticipée de l'activité agricole qui est également envisagée par les autorités de la Communauté européenne, pourrait, de son côté éliminer l'agriculture de montagne. Cette mesure correspond en effet à un système de préretraite avec prime pour les travailleurs agricoles ayant dépassé l'âge de 55 ans. Lorsqu'on sait, par exemple, que $35 \%$ des chefs d'exploitation ont plus de 55 ans en moyenne dans les Pyrénées françaises et qu'ils n'ont pas de successeurs, on peut s'interroger sur l'avenir, d'autant que $11 \%$ seulement ont moins de 35 ans.

Si l'on considère le niveau de formation des agriculteurs exigé par les nouveaux règ'ements de la C.E.E., le retard pris ces dernières années en France semble difficile à rattraper. En 1995, tout jeune agriculteur devrait être titulaire du bacca!auréat technique agricole pour bénéficier de la "dotation jeune agriculteur " pour son installation. En 1987, $8 \%$ des installés en montagne ont ce diplôme (16). Le retard est tel que l'installation des jeunes en montagne relèverait de l'illusion.

\section{Vers une cohérence dans les stratégies de développement agricols et rural.}

Les mesures relatives au retrait des terres sont susceptibles d'apporter un certain nombre de bouleversements dans le paysage agricole actuel, notamment celui des zones défavorisées; mais encore faut-il nuancer les appréciations. Comme avec les quotas de production laitiers, la baisse de la production escomptée risque d'être obtenue par disparitoin des

(16) M. Barrué-Pastor, F. Faissat, M. Viale : D.J.A. et installation agricole en Midi-Pyrénées, Toulouse, Rapport 1988, 42 p., 5 cartes, 1 carte couleur, 22 graphes. 
petits agriculteurs et des exploitations les plus fragiles, notamment celles qui se situent en zones déjà défavorisées; dans ce cas, la conséquence directe serait l'aggravation de la désertification.

En zone de montagne, ce ne sont pas les secteurs où la déprise agricole est la plus forte qui seront affectés, mais plutôt les zones qui ont le mieux résisté, celles qui disposent de bonnes terres de fond de vallée, où les structures foncières sont les plus importantes. Selon la taille des exploitations, la topographie des parcelles, le niveau d'investissement, la chute des revenus, l'âge de l'exploitant et les espoirs de succession, le retrait risque d'être saisi comme une opportunité, notamment par les * héritiers " qui vivent d'un revenu non agricole, comme un moyen de salut pour les agriculteurs en grande difficulté ou ceux qui ont du mal à finir leur activité d'agriculteur. Dans les zones de coteaux, ce sont les terres dune faible productivité, où les bons rendements sont aléatoires, où l'usure du matériel est plus accentuée, où les risques d'accidents ou de perte de récolte sont grands qui risquent d'être les premières à être livrées à la jachère ou au boisement. C'est, sans nul doute, du rapport entre le montant de la prime et celui des charges fixes (et de structures) que dépendra la réalité de l'incitation. Pour le moment, le montant de la prime ne paraît pas suffisamment incitatif.

Quoi qu'il en soit, l'application des mesures qui visent à réduire le volume de la production agricole risque d'élargir les poches de désertification qui se développent ici ou là, soit en détruisant les derniers îlots de résistances de l'agriculture dans les zones à forte déprise, soit en s'attaquant à l'agriculture de coteaux en difficulté, comme c'est le cas en zone pré-pyrénéenne, soit en anéantissant les dernières chances de restructuration foncière; de nombreux héritiers, non agriculteurs, qui ont toujours refusé de vendre ou de signer des baux de fermage pourraient bien trouver dans cette formule une manière de couvrir leurs frais fixes.

Pour les jeunes agriculteurs installés en zone défavorisée, la question principale va demeurer celle du foncier. A celui de la gestion individuelle de la propriété de fond de vallée va s'ajouter le nouvel enjeu de la gestion des territoires communaux, qui prendront de plus en plus d'importance en tant que facteur d'équilibre des exploitations. Tout va dépendre de la capacité des ieunes agriculteurs à s'emparer des structures de gestion collective du foncier, telles les «associations foncières pastorales» et des organes utilisateurs, tels les "groupements pastoraux". Ici encore ce sont les zones qui auront pris le plus de retard dans ce domaine, tels les Pyrénées, qui seront les plus menacées.

Les problèmes soulevés dépassent le cadre d'une réflexion qui ne peut se limiter au développement agricole; dans les zones défavorisées plus qu'ailleurs, son imbrication avec le développement rural et l'ensemble de son environnement micro-régional est total. Cette imbrication a plus de poids que la stricte logique de la filière agricole.

Par ailleurs, même si l'agriculture n'est plus considérée depuis longtemps comme l'unique moteur du développement rural, sa disparition a 
montré à quel point elle restait indispensable en tant que principale gestionnaire de la diversité des espaces. Or, l'avenir du monde rural, la cohésion économique des régions ainsi que la protection de l'environnement et du patrimoine naturel sont de plus en plus liés à la politique agricole de la C.E.E. Une question vive demeure: quelle place et quel intérêt seront accordés aux programmes régionaux de développement rural ?

Les derniers décrets sur le retrait des terres arables ont mis en évidence la triple urgence d'une modulation des mesures européennes prises par secteur d'actıvité en fonction des particularismes régionaux, d'une articulation entre les politiaues sectorielles (ici la po.itique agricole commune) et les politiques globales d'aménagement rural, d'une exclusion de l'ensemb.e des zones aeıavorisées du périmètre concerné par le retrait des terres arables (17).

Monique Barrué-Pastor.

(17) A ce jour, seules les * zones sensibles aux incidences » de quelques départements du Midi de la France sont exclues. (Arrêté du 18 novembre 1988 relatif aux exemptions de zones (zones exemptée de l'application du dispositif national de retrait des terres agricoles) en application de l'article 2 du décret du 18 novembre 1988 : Alpes-de-Haute-Provence, Hautes-Alpes, Alpes-Maritimes, Ardèche, Aude, Bouches-du-Rhône). 